\title{
INTRODUCTION TO AMUSES: AKARI SURVEY WITH A WINDOW OF OPPORTUNITY
}

\author{
J.H. Kim ${ }^{1}$, M. $\operatorname{Im}^{1}$, H.M. Lee ${ }^{2}$, M.G. Lee ${ }^{2}$ and the AMUSES team
}

\begin{abstract}
With advancement of infrared space telescopes during the past decade, infrared wavelength regime has been a focal point to study various properties of galaxies with respect to evolution of galaxies. Polycyclic Aromatic Hydrocarbons (PAHs) have emerged as one of the most important features since these features dominate the midinfrared spectra of galaxies. These PAH features provide a great handle to calibrate star formation rates and diagnose ionized states of grains. However, the PAH $3.3 \mu \mathrm{m}$ feature has not been studied as much as other PAH features since it is weaker than others and resides outside of Spitzer capability, although it will be the only PAH feature accessible by JWST for high-z galaxies. AKARI mJy Unbiased Survey of Extragalactic Sources in 5MUSES (AMUSES) intends to take advantage of AKARI capability of spectroscopy in the $2 \sim 5 \mu \mathrm{m}$ to provide an unbiased library of 44 sample galaxies selected from a parent sample of 5MUSES, one of Spitzer legacy projects. For these $3.6 \mu \mathrm{m}$ flux limited sample galaxies whose redshifts range between $0<z<1$, AMUSES will calibrate PAH $3.3 \mu \mathrm{m}$ as a star formation rate (SFR) indicator while measuring ratios between $\mathrm{PAH}$ features. We present preliminary results of AMUSES.
\end{abstract}

\section{Introduction}

Infrared (IR) astronomy has progressed tremendously over the past decades thanks to various space missions, such as the Infra-Red Astronomical Satellite (IRAS) (Soifer et al. 1987), the Infrared Space Observatory (ISO) (Kessler et al. 1996; Genzel \& Cesarsky 2000), and the Spitzer space telescope (Werner et al. 2004). While providing clues to numerous astrophysical subjects, these space-based observatories also improve extragalactic astronomy since these IR wavelengths carry

\footnotetext{
1 CEOU, Department of Physics and Astronomy, Seoul National University

2 Department of Physics and Astronomy, Seoul National University
} 
a vast information on galaxies. For example, the shortest IR wavelengths (up to $10 \mu \mathrm{m})$ represent the photospheric light and are good stellar mass indicators (Regan \& The Sings Team 2004). Then, dust grains at different temperatures are represented by different ranges of IR wavelengths (Genzel \& Cesarsky 2000). IR wavelengths also carry numerous lines from hydrogen molecule and gas-phase species. However, the most important aspect of IR astronomy on galaxies is probably that IR emission represents dust-obscured star formation activity of galaxies (Genzel \& Cesarsky 2000). While other shorter wavelength star formation rate (SFR) proxies, such as hydrogen recombination lines and ultraviolet (UV) emissions rely on the measurement of photoionizing UV photons from heavy stars and, thus, suffer from extinction, the bolometric IR luminosity measures dust-obscured star formation within galaxies and is less affected by extinction. However using the bolometric IR luminosity as a SF indicator has two caveats. First, not only newly formed heavy stars, but also evolved stellar populations can heat dust components within galaxies ( $c f$. Calzetti elsewhere in this volume). Then it is extremely tricky to understand the whole range of IR spectral energy distribution (SED) for high-z galaxies.

Therefore, many studies have attempted calibrate reliable IR SF proxies to bolometric IR luminosities in recent years. Among these IR SFR proxies, polycyclic aromatic hydrocarbons (PAHs) have gotten enormous attention due to their ubiquity and strong potential as diagnostics of other properties. On the basis of the aromatic IR band emission, PAHs are considered to be present in a wide range of objects and environments, such as post-AGB stars, planetary nebulae, HII regions, reflection nebulae and the diffuse interstellar medium (Puget et al. 1985; Allamandola et al. 1989). The PAH mid-IR features are believed to contribute up to $10 \%$ of the total IR luminosity of star forming galaxies (Helou et al. 2001; Peeters et al. 2002; Smith et al. 2007).

Numerous recent studies measure PAH band fluxes and equivalent widths (EWs) in oder to calibrate these emission features as SFR proxies within the Galactic environments and galaxies at higher redshift. These studies reveal that there exist differences in PAH EWs and $\mathrm{L}_{P A H} / \mathrm{L}_{I R}$ ratios between local values and high redshift ones (Helou et al. 2001; Peeters et al. 2002; Smith et al. 2007). Since PAH band ratios reflect variations in physical conditions within environments, such as ionization states of dust grains and metallicity (Smith et al. 2007; Galliano et al. 2008; Gordon et al. 2008. See papers by Draine and by Galliano elsewhere in this volume), more detailed study on this subject will put a better constraint on physical conditions of PAH emission sites and calibration of PAH bands as SFR proxies.

Then, most studies on PAH emission features concentrate on stronger bands, such as $6.2,7.7$, and $11.3 \mu \mathrm{m}$ due to the relatively weaker strength of $3.3 \mu \mathrm{m}$ feature and lack of wavelength coverage by instruments on board Spitzer. The Japanese space-based IR observatory, AKARI can fill this gap (Murakami et al. 2007). Launched on February 26th, 2006, AKARI has a $68.5 \mathrm{~cm}$ telescope which is cooled down to $65 \mathrm{~K}$. It has two instruments, Far-Infrared Surveyor (FIS) and InfraRed Camera (IRC), whose wavelength coverages range from 1.8 to $180 \mu \mathrm{m}$. 
Developed to carry out all-sky survey, AKARI has two operation modes, survey mode and pointing mode. More details on AKARI, its operation and accomplishments can be found in the literature. Spectroscopic observations of PAHs have also been reported by Onaka et al. elsewhere in this volume.

We present one of AKARI mission projects (MPs), AKARI mJy Unbiased Survey of Extragalactic Sources (AMUSES). The main scientific goal of AMUSES is to construct a continuous spectral library over the wavelength window between 2.5 and $40 \mu \mathrm{m}$ for a subsample of $5 \mathrm{mJy}$ Unbiased Spitzer Extragalactic Survey (5MUSES) (Wu et al., accepted). AMUSES enables to access the $2.5-5 \mu \mathrm{m} \mathrm{spec}-$ tral window which includes the $3.3 \mu \mathrm{m}$ emission feature. Moreover, the $3.3 \mu \mathrm{m}$ $\mathrm{PAH}$ feature is the only dust emission feature at high redshift $(z>4.5)$ accessible to JWST. Therefore, understanding and calibrating the $3.3 \mu \mathrm{m}$ feature with respect to the total IR luminosity, the SFR and other PAH emission features for local galaxies is critical for interpreting JWST spectra of dusty galaxies in future.

\section{Sample selection}

We select our sample from 5MUSES. 5MUSES, one of the Spitzer Legacy surveys, performs a mid-infrared spectroscopic observation of extragalactic sources brighter than $5 \mathrm{mJy}$ at $24 \mu \mathrm{m}$ in the Spitzer First Look Survey (FLS) field and four subfields of Spitzer Wide-area Infrared Extragalactic (SWIRE) survey with the Infrared Spectrograph (IRS) onboard the Spitzer space telescope. The main scientific goal of 5MUSES is to provide an unbiased library of infrared spectra from 5 to $40 \mu \mathrm{m}$ of sources which have not been sought after in previous studies. Since the main objective of AMUSES is to detect the $3.3 \mu \mathrm{m}$ PAH feature, we narrow down our sample to be brighter than $1 \mathrm{mJy}$ at $3.6 \mu \mathrm{m}$. Based on the Spitzer IRAC $3.6 \mu \mathrm{m}$ data for the 5MUSES sample, we find that 60 of the 330 5MUSES galaxies satisfy the flux cut of $1 \mathrm{mJy}$ at $3.6 \mu \mathrm{m}$. In order to detect the $3.3 \mu \mathrm{m}$ feature, we limit the redshift range to $z<0.5$. With this additional cut, we narrow down the sample size to 51 galaxies. In addition to this base sample, we add 10 targets with their $3.6 \mu \mathrm{m}$ flux brighter than $0.7 \mathrm{mJy}$ whose redshifts could not be determined by optical spectroscopy. After this selection, 60 target galaxies remain.

Then 44 targets are approved for the final program. Based on the SED classification, there are 22 AGNs and 17 starburst galaxies. The rest of the sample shows a composite SEDs. The redshift distribution is presented in Figure 1.

\section{Observation and data reduction}

The main goal of AMUSES is to detect the PAH $3.3 \mu \mathrm{m}$ feature with $\mathrm{S} / \mathrm{N}>5$ with IRC (Onaka et al. 2007). IRC is a workhorse for AKARI and has a field of view of roughly $10^{\prime} \times 10^{\prime}$. Pixel scales for three independent cameras range from $1.45^{\prime \prime}$ through $2.5^{\prime \prime}$. Each observation during the pointing mode has a maximum exposure time of ten minutes, but actual exposure times run between six to seven minutes. In order to achieve this $\mathrm{S} / \mathrm{N}$ for sources with $1 \mathrm{mJy}$ at $3.6 \mu \mathrm{m}$, 


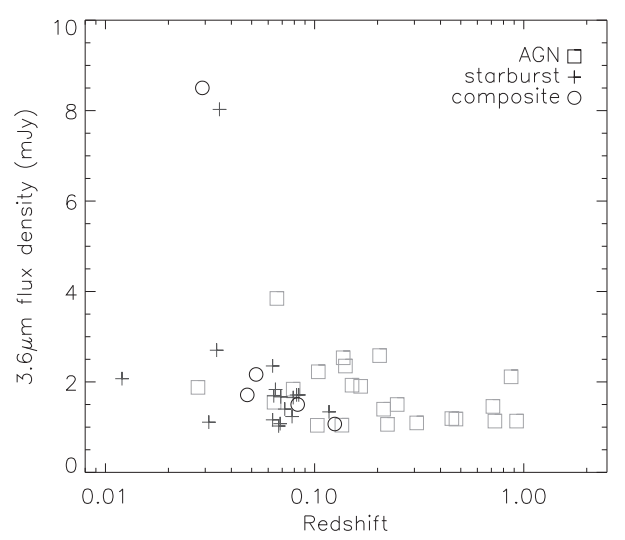

Fig. 1. Redshift distribution of the sample of galaxies of AMUSES plotted against $3.6 \mu \mathrm{m}$ flux. Symbols represent the SED classification of target galaxies based on their MIR colors by 5MUSES collaboration. Open squares represent the AGN-type SED, while crossess represent the starburst-type SED. Open circles represent the composite SED. AGN-type targets are more evenly distributed across the redshift range, while starburst-type targets are more clustered at lower redshifts.

we decided to have three pointings for each target with NIR grism (NG) while adding one pointing with NIR prism (NP) in order to improve continuum extraction. These observations are carried out with a slit aperture whose dimension is $1^{\prime}$ by $1^{\prime}$. In total, 51 pointings are observed for 20 target galaxies. Among them, ten sources have completed their scheduled observations. Data reduction is performed with the IRC spectroscopy pipeline ${ }^{1}$. Additional cosmic ray removal, stacking multipointing exposures, and sigma clipping during stacking are executed individually after running the IRC spectroscopy pipeline. Final one-dimensional spectra are extracted from two-dimensional spectral images which are binned by three pixels along the wavelength direction.

\section{Results}

We present several sample spectra in Figure 2. Overall, the $3.3 \mu \mathrm{m}$ PAH emission feature is pretty weak across the sample even if it is detected. However, it is still noticeable that its strength is stronger for galaxies with starburst SED class than for AGN class. Most galaxies in which the $3.3 \mu \mathrm{m}$ PAH feature is detected with a significant $\mathrm{S} / \mathrm{N}$ are classified as starburst galaxies. The only exceptions are VIIZw353 and 2MASX J16205879+5425127 in which the $3.3 \mu \mathrm{m}$ PAH detection is marginal. The latter is also classified as composite SED.

\footnotetext{
${ }^{1}$ http://www.ir.isas.jaxa.jp/ASTRO-F/Observation/DataReduction/IRC/
} 

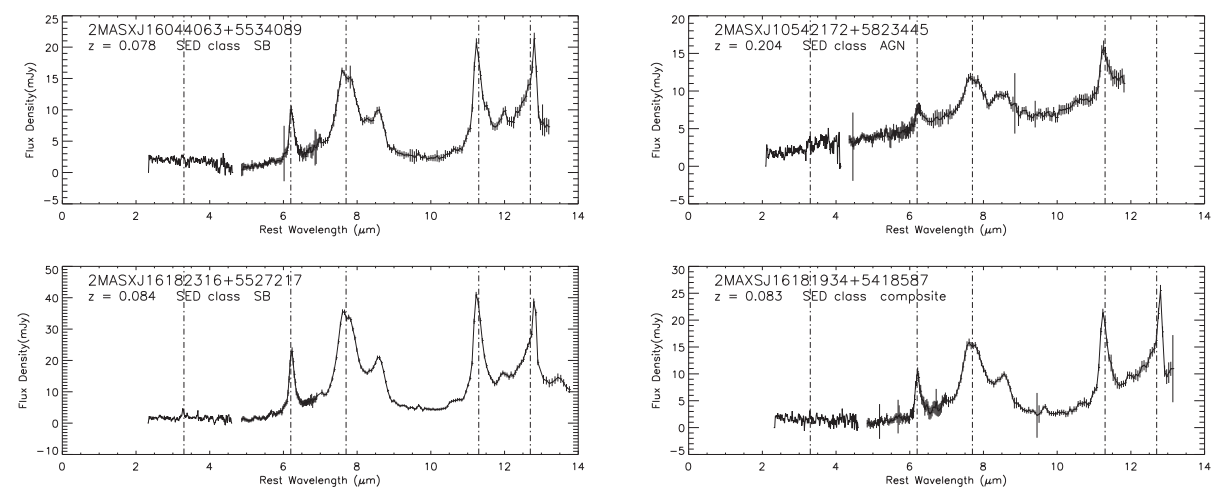

Fig. 2. Sample spectra of AMUSES based on IRC and IRS spectra. Notice the gaps between two instruments around $5 \mu \mathrm{m}$. All spectra are deredshifted. Vertical dashed lines indicate the most notable PAH emission features at 3.3, 6.2, 7.7, 11.2, and $12.7 \mu \mathrm{m}$. The two galaxies on the left panels are classified as starburst galaxies based on their SEDs while the two galaxies on the right panels are classified as AGN, or have a composite SED. The $3.3 \mu \mathrm{m}$ PAH feature is always the weakest and difficult to detect compared to the other prominent PAH emission features.

We also present a couple of plots showing correlations between EWs of the $3.3 \mu \mathrm{m}$, and $6.2 \mu \mathrm{m}$ features (Fig. 3). Bigger symbols represent samples of AMUSES. Asterisks represent galaxies with starburst SEDs while filled circles represent galaxies with AGN SEDs. A diamond represent a galaxy with composite SED. Overplotted are the data from Imanishi et al. (2008). Asterisks represent their samples classified as HII-like galaxies, i.e. star-forming galaxies, while filled circles represent AGNs. Within the sample of Imanishi et al. (2008), two SED classes do not differ much in correlations between EWs of the $3.3 \mu \mathrm{m}$, and $6.2 \mu \mathrm{m}$ bands. They are relatively well matched, albeit with big scatter. AMUSES sample galaxies, most of which are starburst galaxies, have even bigger scatter while generally following the trend set by the sample of Imanishi et al.

\section{Summary}

We introduce a mission project of AKARI, AMUSES and its preliminary results. With AMUSES, we investigate the $3.3 \mu \mathrm{m}$ PAH feature and its correlation with other PAH features as well as other properties of sample galaxies while constructing an unbiased library of sample galaxies selected from a parent sample of 5MUSES. So far, we have measured fluxes and EW of the $3.3 \mu \mathrm{m}$ PAH feature for 20 target galaxies and have compared their EW to the Imanishi et al. (2008) sample. 


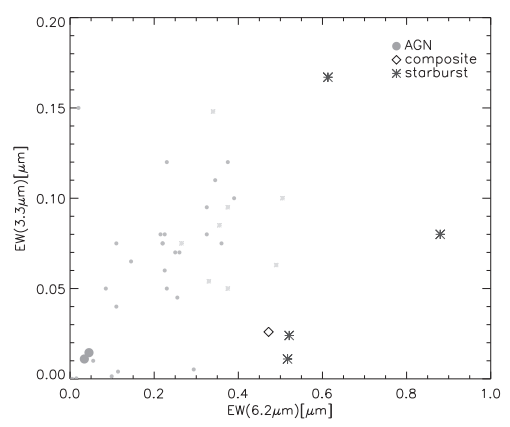

Fig. 3. Correlation between equivalent widths of the $3.3 \mu \mathrm{m}$ PAH line and the $6.2 \mu \mathrm{m}$ $\mathrm{PAH}$ line. Asterisks represent galaxies with starburst SEDs while filled circles represent galaxies with AGN SEDs. A diamond represent a galaxy with composite SED. Overplotted are the data from Imanishi et al. (2008). Their AGN samples are represented by small filled cicles and their HII-like samples are represented by small asterisks.

This work was supported by the National Research Foundation of Korea(NRF) grant funded by the Korean government(MEST), No. 2009-0063616. This research is based on observations with AKARI, a JAXA project with the participation of ESA.

\section{References}

Allamandola, L.J., Tielens, A.G.G.M., \& Barker, J.R., 1989, ApJS, 71, 733

Calzetti, D., et al., 2007, ApJ, 666, 870

Galliano, F., Madden, S.C., Tielens, A.G.G.M., Peeters, E., \& Jones, A.P., 2008, ApJ, 679,310

Genzel, R., \& Cesarsky, C.J., 2000, ARA\&A, 38, 761

Gordon, K.D., Engelbracht, C.W., Rieke, G.H., et al., 2008, ApJ, 682, 336

Helou, G., Malhotra, S., Hollenbach, D.J., Dale, D.A., \& Contursi, A., 2001, ApJ, 548, L73

Imanishi, M., Nakagawa, T., Ohyama, Y., et al., 2008, PASJ, 60, 489

Kessler, M.F., et al., 1996, A\&A, 315, L27

Murakami, H., et al., 2007, PASJ, 59, 369

Onaka, T., et al., 2007, PASJ, 59, 401

Peeters, E., Hony, S., Van Kerckhoven, C., et al., 2002, A\&A, 390, 1089

Puget, J.L., Leger, A., \& Boulanger, F., 1985, A\&A, 142, L19

Regan, M., \& The Sings Team 2004, 35th COSPAR Scientific Assembly, 35, 4098

Smith, J.D.T., et al., 2007, ApJ, 656, 770

Soifer, B.T., Neugebauer, G., \& Houck, J.R., 1987, ARA\&A, 25, 187

Werner, M.W., et al., 2004, ApJS, 154, 1

$\mathrm{Wu}$, Y., et al., 2010, ApJ, accepted 